\title{
BMJ Open Development and use of mobile messaging for individuals with musculoskeletal pain conditions: a scoping review protocol
}

\author{
Simone Scotti Requena, ${ }^{1}$ Michele Sterling, ${ }^{1,2}$ Rachel A Elphinston, ${ }^{1,2,3}$ \\ Carrie Ritchie, ${ }^{1}$ Sarah Robins, ${ }^{1}$ Nigel R Armfield (i) ${ }^{1,4}$
}

To cite: Scotti Requena S, Sterling M, Elphinston RA, et al. Development and use of mobile messaging for individuals with musculoskeletal pain conditions: a scoping review protocol. BMJ Open 2021;11:e048964. doi:10.1136/ bmjopen-2021-048964

\section{- Prepublication history and} additional supplemental material for this paper are available online. To view these files, please visit the journal online (http://dx.doi.org/10.1136/ bmjopen-2021-048964).

Received 12 January 2021 Accepted 29 June 2021

Check for updates

(C) Author(s) (or their employer(s)) 2021. Re-use permitted under CC BY-NC. No commercial re-use. See rights and permissions. Published by BMJ.

${ }^{1}$ RECOVER Injury Research Centre, The University of Queensland, Herston, Queensland, Australia ${ }^{2}$ NHMRC Centre for Research Excellence in Road Traffic Injury Recovery, Herston, Queensland, Australia

${ }^{3}$ School of Psychology, The University of Queensland, Saint Lucia, Queensland, Australia

${ }^{4}$ Centre for Health Services Research, The University of Queensland, Wooloongabba, Queensland, Australia

Correspondence to

Dr Nigel R Armfield;

n.r.armfield@uq.edu.au

\section{ABSTRACT}

Introduction Previous reviews of mobile messaging for individuals with musculoskeletal pain have shown positive effects on pain and disability. However, the configuration of digital content, method of presentation and interaction, dose and frequency needed for optimal results remain unclear. Patient preferences concerning such systems are also unclear. Addressing these knowledge gaps, incorporating evidence from both experimental and observational studies, may be useful to understand the extent of the relevant literature, and to influence the design and outcomes of future messaging systems. We aim to map information that could be influential in the design of future mobile messaging systems for individuals with musculoskeletal pain conditions, and to summarise the findings of efficacy, effectiveness, and economics derived from both experimental and observational studies.

Methods and analysis We will include studies describing the development and/or use of mobile messaging to support adults ( $\geq 18$ years) with acute or chronic musculoskeletal pain. We will exclude digital health studies that lack a mobile messaging component, or those targeted at other health conditions unrelated to the bones, muscles and connective tissues, or involving surgical or patients with cancer, or studies involving solely healthy individuals. Our sources of information will be online databases and reference lists of relevant papers. We will include papers published in English in the last 10 years. Two pairs of independent reviewers will screen, select and extract the data, with any disagreements mediated by a third reviewer. We will report the results according to the Preferred Reporting Items for Systematic Reviews and Meta-Analyses extension for scoping reviews checklist. We will synthesise the findings in a tabular format and provide a descriptive summary.

Ethics and dissemination Formal ethical approval is not required. We will disseminate the findings through publication in a peer-reviewed journal, relevant conferences, and relevant consumer forums.

Trial registration Open Science Framework https://osf.io/ 8mzya; DOI: 10.17605/0SF.I0/8MZYA.

\section{INTRODUCTION}

Consumer mobile devices such as smartphones and tablets have become ubiquitous;
Strengths and limitations of this study

- This review will be the first to map the extent of current knowledge relating to the design and use of mobile messaging interventions for individuals with musculoskeletal pain conditions, incorporating information from both experimental and observational studies.

- This protocol is guided by the Joanna Briggs Institute Manual for Evidence Synthesis guidelines and follows the Preferred Reporting Items for Systematic Reviews and Meta-Analyses extension for scoping reviews.

- Our study is limited to peer-reviewed studies published in the English language, and indexed in four online databases; while our search strategy is broad and covers common relevant health research databases, it is possible that some information of relevance to our review questions will not be captured.

- This study will map the literature; as is common practice with scoping reviews, we will not assess risk of bias, nor the quality of the evidence.

recent surveys have estimated that $88 \%$ of adults in the UK, $91 \%$ in Australia and $97 \%$ in the Middle-East own or have access to a smartphone. ${ }^{1}$ Mobile devices allow both the capture and the display of information, are an inexpensive, convenient and an easyto-use way to provide some health-related services; this use is commonly referred to as 'mHealth'.

mHealth applications may capture data in many ways. For example, the user may be engaged actively through prompts to complete health diaries, or to respond to prompts to complete ecological momentary assessments which involve the repeated collection of individuals' behaviours and experiences in their natural environment. ${ }^{2}$ Built-in cameras can be used to capture still images or short video clips for screening and diagnosis, and passive data about the user's 
movements can be collected using the accelerometer, GPS and from add-on devices such as wearables. Similarly, mHealth applications can deliver information or interventions in many ways: for instance, by providing healthrelated information in plain or multimedia format; ${ }^{3}$; push messages for medication reminders ${ }^{4}$; or ecological momentary interventions. ${ }^{5}$ With the increase in computational power and the availability of the internet, it is possible to create sophisticated mHealth applications that can adapt to the needs of the individual, thus becoming a mechanism to deliver personalised care ${ }^{6}$

mHealth has been applied across the spectrum of health disciplines and across the continuum of care, with examples in health promotion, behaviour change, prevention, ${ }^{7-9}$ screening, ${ }^{10} 11$ diagnosis, ${ }^{12}{ }^{13}$ risk assessment and prognosis, ${ }^{14}{ }^{15}$ therapy, ${ }^{16}{ }^{17}$ monitoring, ${ }^{18}{ }^{19}$ patient self-management, ${ }^{20}{ }^{21}$ and survivorship care. ${ }^{22} 23$ Recent syntheses provide some evidence for the efficacy of mHealth: a 2018 systematic review of systematic reviews (23 reviews; 79665 participants) ${ }^{24}$ and a 2019 metaanalysis (64 studies; 10296 participants) ${ }^{25}$ both reported health outcomes favouring mHealth interventions in chronic disease. Important outcomes included increased physical activity, improvements in the management of asthma, improvements in the symptoms of pulmonary disease and heart failure, better glycaemic control in patients with diabetes, improved management of blood pressure in hypertensive patients, weight reduction in overweight and obese patients and improved adherence to antiretroviral treatments for patients with HIV. Improved health service outcomes included reductions in mortality and hospitalisation, and improved attendance rates. While the evidence so far is encouraging, the authors highlighted that outcomes are mixed, that no long-term studies have yet been reported, and that the methodological quality of included studies was generally low. ${ }^{24} 25$

While there have been many clinically focused reports of mHealth interventions, there have been fewer health economic evaluations, and those that have been conducted have focused on higher-income countries. A 2017 systematic review of economic evaluations of mHealth interventions found that 29 (83\%) of 39 included studies reported that mHealth was cost-effective, provided cost-savings or was otherwise economically beneficial. ${ }^{26}$ The quality of the reporting was described as being of moderate-to-high quality, but because a high proportion of studies reported positive outcomes, the authors cautioned that publication bias may be present. Most of the included studies (27 (69.2\%) of 39) were focused on behaviour change communication, and of those, most (20 $(74.1 \%)$ of 27$)$ showed an economic benefit. Outpatient clinic attendance (eg, reminders to attend) was the largest focus area $(7(17.95 \%)$ of 39$)$ of which $6(85.7 \%)$ of 7 studies showed an economic benefit. Short message service (SMS) was the main communication technique used (22 (56.41\%) of 29) of studies, of which $17(77.3 \%)$ of 22 studies showed an economic benefit. ${ }^{26}$
In this review, we focus on health-related interactions provided by SMS, or by push notifications delivered through applications installed on a mobile device, for individuals with musculoskeletal (MSK) pain conditions. The area has been reviewed previously: a recent systematic review of randomised controlled trials (RCTs) explored the effectiveness of mHealth/digital health for managing MSK conditions. ${ }^{27}$ Five of the 19 included studies (all RCTs; 1086 participants) ${ }^{28-32}$ involved aspects of messaging, with 4 of 5 reporting that the digital interventions were associated with statistically significant improvements in pain ${ }^{29-32}$ and functional disability. ${ }^{28-31}$ A second review (11 RCTs; 1607), focused specifically on the effectiveness of text messaging for the management of MSK pain. ${ }^{33}$ Five studies assessed text messaging as an adjunct to usual care, ${ }^{34-38}$ with improvements found to treatment adherence. In a further five studies, text messaging was assessed as one component of a complex intervention ${ }^{39-43}$ with small but inconsistent effects on pain, functioning, adherence, and quality-of-life. In the remaining study, similar effects on functioning were found when text messaging was compared with telephone counselling. ${ }^{44}$

While systematic reviews have shown positive effects on pain and disability, it is unclear what configuration of digital content, method of presentation, dose and frequency may achieve optimal results. Further, it is unclear what patients may prefer in terms of each of these aspects. Such information may be useful to optimise the design and outcomes achieved by future systems; to identify such information, we must look beyond RCTs.

To our knowledge, no reviews have examined factors that may be important in the design and development of messaging systems for individuals with MSK pain. Similarly, the findings from observational studies of messaging for MSK pain have not been synthesised; consequently, there is a gap in information to guide the development of future messaging systems for this patient group. We conducted a preliminary search of PROSPERO, MEDLINE, the Cochrane Database of Systematic Reviews and Joanna Briggs Institute (JBI) Evidence Synthesis and did not identify any current or underway scoping reviews or systematic reviews with this topic focus.

Our aims are threefold: first, to map how mobile messaging has been used for individuals with MSK pain; second, to identify information that could be useful in the design of future messaging interventions for these individuals; and third, because few RCTs have been conducted in the area, and their focus has been solely on effectiveness, to explore and summarise the findings of efficacy, effectiveness and economics derived from both previous experimental and observational messaging studies for individuals with MSK pain.

\section{Review questions}

1. In the context of MSK pain conditions, for which individuals, with which problems, and for what purpose has messaging on mobile devices been used (eg, 
medication reminders, alert, education, motivation, prevention, and data collection)?

2. What information exists to guide the development of mobile messaging for MSK conditions (eg, frequency of texts, length of texts, duration of intervention, and theoretical basis)?

3. How have patients' preferences been included in the design of a study, and how have their preferences been assessed?

4. What methods have been used to evaluate the use of mobile messaging for MSK conditions (eg, how were outcomes assessed; what processes were involved)?

5. Does the literature support the efficacy, effectiveness, and economics of messaging on mobile devices for individuals with MSK conditions?

\section{Inclusion criteria}

\section{Participants}

We will include studies involving adults ( $\geq 18$ years) experiencing acute or chronic MSK condition (eg, back pain, neck pain, arthritis and osteoarthritis). The term 'MSK conditions' describes those conditions that affect muscles, bones, joints and related tissues. ${ }^{45}$ MSK conditions comprise over 150 diagnoses, ${ }^{45}$ and in 2017 , it was the highest contributor to global disability (as measured by the years lived with disability). ${ }^{45} 46$

We will exclude studies concerning individuals with spinal cord injury, mild traumatic brain injury and moderate-to-severe orthopaedic injuries, and conditions relating to mobile phone overuse. We will also exclude studies targeted at other health conditions unrelated to the bones, muscles and connective tissues (eg, diabetes, asthma, cancer and stroke), studies involving surgical patients, or studies involving solely healthy individuals.

\section{Concept}

We aim to map papers that describe patient-focused, health-related messaging provided on consumer mobile devices such as mobile phones, tablets, personal digital assistants and wearables. Specifically, we will include: (1) papers describing the development and/or evaluation of mobile messaging to support the target population. All types of support will be included (eg, medication reminders, education, motivational messaging, harm prevention and data collection); (2) papers describing messaging delivered by any of the following methods: push notifications arising from mobile applications, SMS or multimedia messaging service (MMS); and (3) studies that involved messaging as an adjunct to an intervention (eg, a psychological intervention combined with SMS reminders or a physiotherapy intervention delivered via an application which included notification reminders).

We will exclude papers that describe the use of other platforms, such as web-based systems or email messages, but that lack any mobile messaging component. We will also exclude studies that provide support other than by mobile messaging support (eg, telephone counselling or reminders by email).
Context

We will include papers in any contextual setting (eg, healthcare facility, hospital, workplace, or university) and conducted in any country.

For studies describing the development of relevant messaging applications, we will include reviews and papers describing experimental/observational/qualitative studies of messaging applications irrespective of study design. For evaluation studies, we will include papers, including reviews, describing economic studies of mobile messaging to support the target population.

We will exclude conference abstracts and editorials because they provide only limited information. We will exclude protocols because they describe proposed work rather than results. While it may be beneficial to include searches of the grey literature, dissertations and literature in languages other than English, doing so would be beyond the resources available to this project. Therefore, our focus is to map what has been published in the peer-reviewed literature, as indexed in the main medical, nursing, allied health and psychology electronic databases.

\section{METHODS AND ANALYSIS}

We developed this protocol using the JBI Manual for Evidence Synthesis, ${ }^{47}$ Section 11.2 for protocol development $^{48}$ and the Preferred Reporting Items for Systematic Reviews and Meta-Analyses extension for scoping reviews (PRISMA-ScR). ${ }^{49}$ This protocol has been registered with the Open Science Framework (https://osf.io/8mzya; DOI: 10.17605/OSF.IO/8MZYA; registered 19 October 2020).

\section{Search strategy}

We have completed electronic database searches using PubMed, CINAHL (via EBSCOhost), Embase and PsycINFO (via APA PsycNET); all searches were conducted on 6 August 2020. We used search strings that described messaging and MSK concepts. We also manually searched the reference lists of relevant papers. We first developed the search strategy for PubMed and subsequently translated it for the other three databases (online supplemental tables S1-S4). Because of resource limitations, we included only papers published in the English language. Finally, because the field of digital health and the capabilities of consumer technology change rapidly, we limited our search to include only papers published in the last 10 years, thus excluding older papers that may not be relevant to contemporary technology and its use.

\section{Screening, inclusion and exclusion process}

We will export the results from the electronic database searches to reference management software (Endnote V.X9) and then subsequently export to Covidence ${ }^{50}$ for further processing. There will be three selection stages: in the first stage, two independent reviewers will screen all titles and abstracts. In the second stage, two pairs of independent reviewers will assess the full-text of eligible papers. A third reviewer will mediate disagreements as 


\begin{tabular}{|c|c|}
\hline Data & Description \\
\hline Authors & Lead author \\
\hline Country & Where the study was conducted \\
\hline Population & Targeted musculoskeletal pain condition \\
\hline Delivery mode & Messaging delivery mode (eg, reminder or notification via a mobile application, SMS and MMS) \\
\hline Messaging characteristics & $\begin{array}{l}\text { Frequency of messaging (eg, random, twice a day or weekly) and numbers of characters of text } \\
\text { messages }\end{array}$ \\
\hline Messaging duration & Duration of messaging intervention (eg, 1-4 weeks or 1 year) \\
\hline Study aims & $\begin{array}{l}\text { What the study aimed to do (eg, improve adherence to medication, improve attendance to } \\
\text { appointments or educate patients on risk factors) }\end{array}$ \\
\hline Theory & If applicable, the theoretical basis of the study (eg, Health Belief Model for behaviour change studies) \\
\hline $\begin{array}{l}\text { Messaging as an additional } \\
\text { support }\end{array}$ & $\begin{array}{l}\text { If applicable, whether mobile messages were used as additional support to the main intervention } \mathrm{Y} / \mathrm{N} \\
\text { (eg, psychological intervention combined with SMS reminders) }\end{array}$ \\
\hline Findings & $\begin{array}{l}\text { If applicable, description of whether the findings support messaging, do not support messaging or are } \\
\text { equivocal }(+/-/=)\end{array}$ \\
\hline Patients' preferences & $\begin{array}{l}\text { If applicable, describe any information relating to patients' preferences (eg, the way patients' } \\
\text { preferences were included in the design and how they were assessed during the implementation } \\
\text { stage) }\end{array}$ \\
\hline
\end{tabular}

MMS, multimedia messaging service; SMS, short message service.

needed. In the final stage, we will manually search for additional papers in the included papers' reference lists.

\section{Data extraction}

Two independent authors will extract the data from each selected study; we created a draft data extraction form (table 1). We will revise this draft as needed after two team members have trialled it with a sample of five included studies. We will resolve any disagreements through discussion, and if a consensus cannot be reached, a third author will mediate until a consensus is met. Where appropriate, we will contact the authors of studies to obtain missing information.

\section{Reporting of results}

First, we will describe the results of our searches and selection process in a PRISMA flow diagram, ${ }^{51}$ reporting the findings according to the PRISMA-ScR checklist. ${ }^{49}$ Second, we will map the available knowledge in a tabular format by the research question (online supplemental tables S5-S7; adapted from the JBI manual ${ }^{48}$ and according to the paper's purpose: (1) papers describing aspects of the development of messaging systems (online supplemental table S6) and (2) studies of the evaluation of messaging systems (online supplemental table S6).

Online supplemental table S5 will tabulate the main characteristics of the included literature as follows: primary author's name, the country where the study was conducted, the design of the study, the primary MSK pain focus of the study (eg, neck, back), study aim(s) (eg, to provide information, behaviour change, data collection and/or development of messaging systems), messaging method (eg, SMS/MMS, app push) and whether messaging was used as an adjunct to another intervention.

Online supplemental table S6 will tabulate the results of the literature relating to aspects of the development of messaging systems. This table will contain information related to messaging content (ie, the format of messaging, eg, text, images, video), theoretical framework, whether the system provided any adaptivity of content according to perceived needs of the user, and messaging dose and behaviour (ie, message frequency, length, timing and adaptivity), measures collected (type of measure, eg, a visual analogue pain score and method of collection) and user experience (eg, any results relating to acceptability, usability, and user preferences).

Online supplemental table S7 will tabulate the results relating to evaluation studies of messaging systems for people with MSK pain. This table will contain information about: outcomes assessed (ie, pain, disability, psychological measures, economics, experience), the primary endpoint (eg, pain in the last week, measured at 6 months postintervention), duration of the intervention and summary findings (whether the findings favour the digital intervention, favour control or are equivocal). The results tables 
may be further refined during the synthesis process by the team members, as per the JBI manual. ${ }^{48}$ Finally, we will provide a narrative summary of the mapped evidence in order to address our aims and research questions.

\section{Patient and public involvement}

It was not appropriate or possible to involve patients or the public in the design, or conduct, or reporting, or dissemination plans of our research.

\section{DISCUSSION}

This review will be the first to map the extent of current knowledge relating to the design and use of mobile messaging interventions for individuals with MSK pain conditions, incorporating information from both experimental and observational studies. This protocol is guided by the JBI Manual for Evidence Synthesis guidelines and follows the PRISMA extension for scoping reviews.

The evidence from experimental studies of the effectiveness of text messaging interventions for managing MSK pain conditions has recently been comprehensively synthesised. ${ }^{33}$ We will build on this work by describing studies relating to the design of messaging systems for MSK conditions, and by synthesising the results from both experimental and observational studies. Because we aim to map the literature, as is common practice with scoping reviews, we will not assess the methodological quality or risk of bias of included studies. ${ }^{49}$ Further, the quality of the evidence of relevant experimental studies has already recently been critically appraised. ${ }^{33}$

Our study is limited to peer-reviewed studies published in the English language and indexed in four online databases. We anticipate that information of most relevance to our questions will be contained in the peer-reviewed literature. While a broader approach may be desirable, we have insufficient resources to translate articles from languages other than English or to examine the grey literature. While our search strategy is broad and covers common relevant health research databases, we acknowledge that it is possible that some information of relevance to our review questions may not be captured.

\section{ETHICS AND DISSEMINATION}

As a review of the available literature, this study does not require formal ethical approval. We will disseminate the findings through publication in a peer-reviewed journal, relevant conferences and relevant consumer forums.

Acknowledgements We would like to acknowledge the contribution of Jackie Devenish, librarian at The University of Queensland, Australia, for assistance in developing the search strategy.

Contributors MS, NRA and SSR conceptualised the study, and SSR developed the initial protocol draft. MS, RAE, CR, SR and NRA contributed substantially to the further development of the draft. NRA and SSR conducted revisions resulting from the peer-review process. All authors reviewed and approved the final draft. SSR and NRA developed the search strategy. MS, RAE, CR and SR critically reviewed the search strategy and provided refinements. SSR conducted the literature searches. SSR, NRA, CR and SR are conducting the screening. SSR and NRA will extract data. All authors will contribute to the reporting of the review described in this manuscript.

Funding The authors have not declared a specific grant for this research from any funding agency in the public, commercial or not-for-profit sectors.

Competing interests None declared.

Patient consent for publication Not required.

Provenance and peer review Not commissioned; externally peer reviewed.

Supplemental material This content has been supplied by the author(s). It has not been vetted by BMJ Publishing Group Limited (BMJ) and may not have been peer-reviewed. Any opinions or recommendations discussed are solely those of the author(s) and are not endorsed by BMJ. BMJ disclaims all liability and responsibility arising from any reliance placed on the content. Where the content includes any translated material, BMJ does not warrant the accuracy and reliability of the translations (including but not limited to local regulations, clinical guidelines, terminology, drug names and drug dosages), and is not responsible for any error and/or omissions arising from translation and adaptation or otherwise.

Open access This is an open access article distributed in accordance with the Creative Commons Attribution Non Commercial (CC BY-NC 4.0) license, which permits others to distribute, remix, adapt, build upon this work non-commercially, and license their derivative works on different terms, provided the original work is properly cited, appropriate credit is given, any changes made indicated, and the use is non-commercial. See: http://creativecommons.org/licenses/by-nc/4.0/.

\section{ORCID iD}

Nigel R Armfield http://orcid.org/0000-0003-4218-0030

\section{REFERENCES}

1 Deloitte LLP. Deloitte's 2019 Global Mobile Consumer Survey, 2019. Available: https://www2.deloitte.com/us/en/insights/industry/ telecommunications/global-mobile-consumer-survey.html [Accessed 27 Jul 2020].

2 Shiffman S, Stone AA, Hufford MR. Ecological momentary assessment. Annu Rev Clin Psychol 2008;4:1-32.

3 Timmers T, Janssen L, Kool RB, et al. Educating patients by providing timely information using smartphone and tablet apps: systematic review. J Med Internet Res 2020;22:e17342.

4 Tabi K, Randhawa AS, Choi F, et al. Mobile apps for medication management: review and analysis. JMIR Mhealth Uhealth 2019;7:e13608.

5 Marciniak MA, Shanahan L, Rohde J, et al. Standalone smartphone cognitive behavioral Therapy-Based ecological Momentary interventions to increase mental health: narrative review. JMIR Mhealth Uhealth 2020;8:e19836.

6 Shaw RJ, Bonnet JP, Modarai F, et al. Mobile health technology for personalized primary care medicine. Am J Med 2015;128:555-7.

7 Kampmeijer R, Pavlova M, Tambor M, et al. The use of e-health and $\mathrm{m}$-health tools in health promotion and primary prevention among older adults: a systematic literature review. BMC Health Serv Res 2016;16 Suppl 5:290.

8 Melia R, Francis K, Hickey E, et al. Mobile health technology interventions for suicide prevention: systematic review. JMIR Mhealth Uhealth 2020;8:e12516.

9 Mayo Clin Proc. Digital health interventions for the prevention of cardiovascular disease: a systematic review and meta-analysis. Elsevier, 2015.

10 Swanepoel DW, Myburgh HC, Howe DM, et al. Smartphone hearing screening with integrated quality control and data management. Int $J$ Audiol 2014;53:841-9.

11 Haberman ZC, Jahn RT, Bose R, et al. Wireless smartphone ECG enables large-scale screening in diverse populations. J Cardiovasc Electrophysiol 2015;26:520-6.

12 Freeman K, Dinnes J, Chuchu N, et al. Algorithm based smartphone apps to assess risk of skin cancer in adults: systematic review of diagnostic accuracy studies. BMJ2020;24:m127.

13 Tatum WO, Hirsch LJ, Gelfand MA, et al. Assessment of the predictive value of outpatient smartphone videos for diagnosis of epileptic seizures. JAMA Neurol 2020;77:593-600.

14 Roeing KL, Hsieh KL, Sosnoff JJ. A systematic review of balance and fall risk assessments with mobile phone technology. Arch Gerontol Geriatr 2017;73:222-6.

15 Szanto Z, Benko I, Jakab L, et al. The use of a smartphone application for fast lung cancer risk assessment†. Eur J CardioThorac Surg 2017;51:1171-6. 
16 Fisher E, Law E, Dudeney J, et al. Psychological therapies (remotely delivered) for the management of chronic and recurrent pain in children and adolescents. Cochrane Database Syst Rev 2019;4:CD011118.

17 Huckvale K, Nicholas J, Torous J, et al. Smartphone apps for the treatment of mental health conditions: status and considerations. Curr Opin Psychol 2020;36:65-70.

18 Chernetsky Tejedor S, Sharma J, Lavallee DC, et al. Identification of important features in mobile health applications for surgical site infection surveillance. Surg Infect 2019;20:530-4.

19 Dogan E, Sander C, Wagner X, et al. Smartphone-Based monitoring of objective and subjective data in affective disorders: where are we and where are we going? Systematic review. J Med Internet Res 2017;19:e262.

20 Whitehead L, Seaton P. The effectiveness of self-management mobile phone and tablet Apps in long-term condition management: a systematic review. J Med Internet Res 2016;18:e97.

21 Marcano Belisario JS, Huckvale K, Greenfield G, et al. Smartphone and tablet self management apps for asthma. Cochrane Database Syst Rev 2013;2013:Cd010013.

22 Hernandez Silva E, Lawler S, Langbecker D. The effectiveness of mHealth for self-management in improving pain, psychological distress, fatigue, and sleep in cancer survivors: a systematic review. J Cancer Surviv 2019;13:97-107.

23 Vollmer Dahlke D, Fair K, Hong YA, et al. Apps seeking theories: results of a study on the use of health behavior change theories in cancer survivorship mobile apps. JMIR Mhealth Uhealth 2015;3:e31.

24 Marcolino MS, Oliveira JAQ, D'Agostino M, et al. The impact of mHealth interventions: systematic review of systematic reviews. JMIR Mhealth Uhealth 2018;6:e23.

25 Yang Q, Van Stee SK. The comparative effectiveness of mobile phone interventions in improving health outcomes: meta-analytic review. JMIR Mhealth Uhealth 2019;7:e11244.

26 Iribarren SJ, Cato K, Falzon L, et al. What is the economic evidence for mHealth? A systematic review of economic evaluations of mHealth solutions. PLoS One 2017;12:e0170581.

27 Hewitt S, Sephton R, Yeowell G. The effectiveness of digital health interventions in the management of musculoskeletal conditions: systematic literature review. J Med Internet Res 2020;22:e15617.

28 Chhabra HS, Sharma S, Verma S. Smartphone APP in selfmanagement of chronic low back pain: a randomized controlled trial. Eur Spine J 2018;27:2862-74.

29 Irvine AB, Russell H, Manocchia M, et al. Mobile-Web APP to selfmanage low back pain: randomized controlled trial. J Med Internet Res 2015;17:e1.

30 Mecklenburg G, Smittenaar P, Erhart-Hledik JC, et al. Effects of a 12-week digital care program for chronic knee pain on pain, mobility, and surgery risk: randomized controlled trial. J Med Internet Res 2018;20:e156.

31 Shebib R, Bailey JF, Smittenaar P, et al. Randomized controlled tria of a 12-week digital care program in improving low back pain. $n p$ j Digital Med 2019;2:1-8.

32 Toelle TR, Utpadel-Fischler DA, Haas K-K, et al. App-based multidisciplinary back pain treatment versus combined physiotherapy plus online education: a randomized controlled trial. NPJ Digit Med 2019;2:1-9.

33 Fritsch CG, Ferreira PH, Prior JL, et al. Effects of using text message interventions for the management of musculoskeletal pain: a systematic review. Pain 2020;161:2462-75.

34 Brix LD, Bjørnholdt KT, Thillemann TM, et al. The effect of text messaging on medication adherence after outpatient knee arthroscopy: a randomized controlled trial. J Perianesth Nurs 2019;34:710-6.
35 Chen H-C, Chuang T-Y, Lin P-C, et al. Effects of messages delivered by mobile phone on increasing compliance with shoulder exercises among patients with a frozen shoulder. J Nurs Scholarsh 2017:49:429-37.

36 Kuusalo L, Sokka-Isler T, Kautiainen H, et al. Automated text Message-Enhanced monitoring versus routine monitoring in early rheumatoid arthritis: a randomized trial. Arthritis Care Res 2020;72:319-25.

37 Mary A, Boursier A, Desailly Henry I, et al. Mobile phone text messages and effect on treatment adherence in patients taking methotrexate for rheumatoid arthritis: a randomized pilot study. Arthritis Care Res 2019;71:1344-52.

38 Smith OJ, Stewart CJ, Rastogi N, et al. Evaluation of the effect of an innovative automated text messaging service on patient experience in day-case hand trauma surgery. J Plast Reconstr Aesthet Surg 2018;71:450-1.

39 Campbell KJ, Louie PK, Bohl DD, et al. A novel, automated TextMessaging system is effective in patients undergoing total joint arthroplasty. J Bone Joint Surg Am 2019;101:145-51.

40 Kristjánsdóttir Ólöf Birna, Fors EA, Eide E, et al. A smartphonebased intervention with diaries and therapist feedback to reduce catastrophizing and increase functioning in women with chronic widespread pain. Part 2: 11-month follow-up results of a randomized trial. J Med Internet Res 2013;15:e72.

41 Lambert TE, Harvey LA, Avdalis C, et al. An APP with remote support achieves better adherence to home exercise programs than paper handouts in people with musculoskeletal conditions: a randomised trial. J Physiother 2017;63:161-7.

42 Thomsen T, Aadahl M, Beyer N, et al. The efficacy of motivational counselling and SMS reminders on daily sitting time in patients with rheumatoid arthritis: a randomised controlled trial. Ann Rheum Dis 2017;76:1603-6.

43 Wang Y, Lombard C, Hussain SM, et al. Effect of a low-intensity, selfmanagement lifestyle intervention on knee pain in community-based young to middle-aged rural women: a cluster randomised controlled trial. Arthritis Res Ther 2018;20:74.

44 Park KH, Song MR. The effects of Postdischarge telephone counseling and short message service on the knee function, activities of daily living, and life satisfaction of patients undergoing total knee replacement. Orthop Nurs 2017;36:229-36.

45 World Health Organization. Musculoskeletal conditions, 2019. Available: https://www.who.int/news-room/fact-sheets/detail/ musculoskeletal-conditions [Accessed 11 June 2020].

46 Institute for Health Metrics and Evaluation. Global burden of disease study 2017. Seattle, United States: Institute for Health Metrics and Evaluation (IHME), The University of Washington, 2017. https:// vizhub.healthdata.org/gbd-compare/

47 Peters MDJ, Marnie C, Tricco AC, et al. Updated methodological guidance for the conduct of scoping reviews. JBI Evid Synth 2020;18:2119-26.

48 Peters M, Godfrey C, Mclnerney P. Chapter 11: Scoping Reviews (2020 version). In: Aromataris E, Munn Z, eds. JBI Manual for Evidence Synthesis [Internet. Adelaide: JBI, 2020.

49 Tricco AC, Lillie E, Zarin W, et al. PRISMA extension for scoping reviews (PRISMA-ScR): checklist and explanation. Ann Intern Med 2018;169:467-73.

50 Veritas Health Innovation. Covidence systematic review software. Melbourne, Australia.

51 Moher D, Liberati A, Tetzlaff J, et al. Preferred reporting items for systematic reviews and meta-analyses: the PRISMA statement. PLoS Med 2009;6:e1000097. 